\title{
Efecto derrame del crecimiento de China en América del Sur: un análisis basado en el comercio internacional
}

\author{
Gercione Dionizio Silva, Marília Fernandes Maciel Gomes \\ y Evandro Camargos Teixeira
}

\section{Resumen}

Dado el aumento de la participación de China en la agenda comercial de América del Sur desde 2001, el crecimiento de aquel país adquirió una enorme importancia para las economías sudamericanas. Se procura analizar el efecto derrame del crecimiento de China en el crecimiento de las principales economías sudamericanas (Argentina, Brasil, Chile y Colombia) entre 1981 y 2014. Se analiza si el aumento de la participación china en la agenda comercial de estos países a partir de 2001 ha alterado dicho efecto. Este efecto es positivo, si bien no se han producido cambios significativos al respecto. La expansión de las exportaciones de los sectores tradicionales de las economías sudamericanas (productos básicos) no resulta suficiente para ampliar las ganancias con el crecimiento de China. Se destaca la importancia de las políticas públicas que diversifiquen la cartera de exportaciones sudamericanas hacia China, como los incentivos a las exportaciones de sectores no tradicionales.

\section{Palabras clave}

China, comercio internacional, relaciones económicas, crecimiento económico, América del Sur, exportaciones, medición, modelos econométricos

\section{Clasificación JEL}

E12, F15, F4

\section{Autores}

Gercione Dionizzio Silva es Doctorando del Programa de posgrado en Economía aplicada en el Departamento de Economía Rural de la Universidad Federal de Viciçosa (Brasil). Correo electrónico: gercione.silva@ufv.br.

Marília Fernandes Maciel Gomes es Profesora Jubilada del Departamento de Economía Rural de la Universidad Federal de Viciçosa (Brasil). Correo electrónico: mariliamacielgomes@gmail.com.

Evandro Camargos Teixeira es Profesor Adjunto Grado IV en el Departamento de Economía de la Universidad Federal de Viciçosa (Brasil). Correo electrónico: evandro.texeira@ufv.br. 


\section{Introducción}

En las últimas décadas el proceso de integración comercial internacional ha avanzado de forma rápida y heterogénea, unificando los espacios económicos de países con distintos niveles de desarrollo. En un espacio económico integrado a nivel internacional, las variaciones en la coyuntura económica de un país pueden afectar el desempeño y el bienestar de otras economías con las que este país está integrado (Dornbusch, 1976; Obstfeld y Rogoff, 1995).

Debido a los lazos comerciales existentes entre los países, derivados principalmente del comercio intersectorial, la demanda extranjera de productos nacionales adquiere gran importancia para las actividades industriales internas. Desde esta perspectiva, los responsables de la formulación de políticas y los equipos de gestión de las grandes empresas también han observado de cerca las actividades económicas de países y agrupaciones económicas importantes como los Estados Unidos, la Unión Europea y China.

En concreto, se ha prestado gran atención al impacto que el notable crecimiento de China ha tenido en el escenario internacional en los últimos años. China se ha destacado en el mercado internacional no solo por ser uno de los principales países emergentes, sino también uno de los principales polos comerciales del mundo. Ya en 2013 el país representaba un promedio del 11\% de las exportaciones e importaciones de productos, así como del 4,4\% de las exportaciones y del 7,5\% de las importaciones de servicios a nivel mundial (OMC, 2015).

A través de sus relaciones de compra y venta de productos, China desempeña un papel crucial en los flujos comerciales de varios países y, en especial, en el comercio de América del Sur. Aunque no se trata de un socio comercial reciente, el comercio de China con este subcontinente comenzó a destacarse a partir de 2001. En términos de flujo comercial, se aprecia a partir de ese período un incremento exponencial de las exportaciones de los principales países de América del Sur (Argentina, Brasil, Chile y Colombia) hacia China.

Entre 1995 y 2000, el crecimiento de las exportaciones sudamericanas hacia China fue de aproximadamente el 55\%, lo que representó un incremento en el valor exportado de casi mil millones de dólares. Entre 2001 y 2014, el incremento fue del 1.595\%. En este período los valores exportados a China pasaron de aproximadamente 4.000 millones de dólares en 2001 hasta aproximadamente 69.000 millones de dólares en 2014. El crecimiento de las importaciones de productos chinos en América del Sur presentó un comportamiento similar. Entre 1995 y 2000 el aumento de las importaciones fue de un 140\%, mientras que de 2001 a 2014 fue de aproximadamente un $1.830 \%$ (UNCTAD, 2015).

Debido a su inserción en los mercados sudamericanos, a partir de 2001 China se ha convertido en uno de los principales socios comerciales de América del Sur. Por lo que respecta a la participación en la agenda comercial de América del Sur, solo los Estados Unidos y la Unión Europea alcanzan niveles similares a China.

Cabe destacar que el significativo aumento de las exportaciones sudamericanas hacia China es ante todo consecuencia del continuo crecimiento que este país ha venido experimentando en las últimas décadas. Según datos de la UNCTAD (2015), el producto interno bruto (PIB) chino ha crecido una media de 9,86 puntos porcentuales por año desde 1981. Se trata de un valor notablemente distinto al de otros países con un nivel de desarrollo similar. Por ejemplo, la tasa media de crecimiento que presentaron los países en desarrollo en este mismo período fue de aproximadamente 4,74 puntos porcentuales, y de 6,74 puntos en el caso de los BRICS. Por consiguiente, el desempeño económico de China y sus políticas externas han pasado a ejercer gran influencia en el crecimiento de los países de América del Sur. 
Si destacamos solo el efecto del crecimiento chino sobre las exportaciones sudamericanas, se puede inferir que este influyó de manera positiva sobre el crecimiento de América del Sur y que este efecto fue aún mayor a partir de 2001. A esta conclusión se llega a partir del aumento de la participación china en la demanda internacional de productos sudamericanos, es decir, en las exportaciones sudamericanas. En otras palabras, se puede inferir que el efecto derrame (spillover $)^{1}$ del crecimiento chino sobre las economías de América del Sur fue positivo y aumentó a partir de 2001.

Sin embargo, el patrón de comercio entre estos países pone en evidencia algunos puntos que deben ser cuestionados. En primer lugar, se observa que existe una gran disparidad en términos de valor agregado entre lo que se exporta hacia China y lo que se importa desde allí. Según Cunha (2011), las transacciones de los países de América del Sur con sus socios comerciales se centran principalmente en la exportación de productos de bajo valor agregado y en la importación de productos de elevado valor agregado. A partir de un análisis del Brasil, principal representante de los países de América del Sur por lo que respecta al comercio, Mattos y Carcanholo (2012) resaltan que, históricamente, la expansión de las exportaciones desde el Brasil hacia China se basa en productos de bajo valor agregado. Por su parte, el aumento de las importaciones ha correspondido principalmente a productos de un mayor valor agregado.

En segundo lugar, también cabe destacar que la diversidad de la cartera de exportaciones sudamericanas a China no ha sufrido grandes cambios en las últimas décadas. Sobre la base del índice de complementariedad elaborado por Michaely ${ }^{2}$, se comprueba que existe una baja complementariedad comercial entre estos países. Entre 1995 y 2013, las exportaciones de América del Sur respondieron en promedio solo al 29,1\% del total de productos importados por China. Por su parte, las exportaciones de China respondieron aproximadamente al $44,9 \%$ de la demanda de las economías sudamericanas.

A pesar de su sencillez, lo anterior pone de manifiesto que puede existir una limitación en las potenciales ganancias para América del Sur procedentes del crecimiento de China. Pese a la importancia que reviste estimular el incremento de las exportaciones de productos tradicionales en la cartera de exportaciones de América del Sur, puede que ello no sea suficiente para impulsar el efecto derrame del crecimiento chino.

A partir de estos planteamientos, el objetivo del presente trabajo es analizar el efecto derrame del crecimiento de China sobre la tasa de crecimiento de los países sudamericanos en el período 1981-2014. A través de este análisis se pretende comprobar si el aumento significativo de la participación china en la agenda comercial sudamericana a partir de 2001 ha modificado el efecto derrame del crecimiento chino. Con los resultados de este análisis se pretende dar respuesta a la siguiente pregunta: ¿la ampliación a China de las exportaciones sudamericanas de sectores ya tradicionales (productos básicos) es suficiente para que estos países se beneficien del crecimiento de China?

Además de la presente introducción, este artículo se divide en cuatro secciones. La siguiente sección presenta un breve análisis de la literatura sobre la importancia del comercio exterior para el crecimiento de una economía, destacando los vínculos existentes en el crecimiento de dos economías. La tercera sección aborda los aspectos metodológicos empleados en este estudio. Los resultados obtenidos se detallan en el apartado cuarto y, por último, en la quinta sección se presentan las consideraciones finales.

\footnotetext{
1 Término empleado para referirse al trasvase del crecimiento de una economía a otra (Poirson y Weber, 2011).

2 El índice de complementariedad de Michaely (1996) indica en qué medida las exportaciones de un país o grupo se corresponden a las importaciones de otro. Este índice va de 0 a 1, donde 0 indica que no existe correspondencia y 1 una correspondencia perfecta.
} 


\section{Revisión de la literatura}

En la literatura sobre el tema, el impacto que el crecimiento económico de un país ejerce sobre otro país o conjunto de países se denomina derrame de crecimiento. Estudios como los de Poirson y Weber (2011), Bayoumi y Swiston (2009) y Helbling y otros (2007) destacan el carácter positivo del efecto derrame. Sin embargo, estos trabajos limitan sus análisis principalmente a los países con un mayor desarrollo industrial (economías desarrolladas) y se centran en casos como los de Estados Unidos, el Japón, la eurozona y Alemania.

Es importante destacar que el efecto de este derrame depende del nivel de desarrollo de las economías, del nivel de integración y del canal de transmisión (Grossman y Helpman, 1997; Poirson y Weber, 2011; Helbling y otros, 2007). Según Bayoumi y Swiston (2009), los tres principales canales de transmisión de este efecto son los intercambios internacionales, los mercados financieros y los precios de los productos básicos. El presente trabajo se basa en los intercambios internacionales como canal de propagación del efecto derrame del crecimiento de China. No obstante, dada la ambigüedad de los efectos del comercio sobre el crecimiento de una economía, es preciso destacar algunos puntos.

Comúnmente, incluso con la presencia de este efecto ambiguo del comercio internacional, las investigaciones acerca del efecto derrame del crecimiento destacan su carácter positivo, dado que el crecimiento de la renta de un país estimulará las importaciones de los productos de un socio comercial. Sin embargo, tomando principalmente como base los intercambios internacionales como canal de transmisión, cabe destacar que el efecto derrame del crecimiento depende ante todo de la agenda comercial entre los países. El aumento de la productividad de un país puede ocasionar un aumento de la demanda de productos de un socio comercial, es decir, incrementar las exportaciones de dicho socio. No obstante, este aumento puede elevar también las importaciones de dicho socio comercial como consecuencia de la mayor competitividad de los productos.

Desde esta perspectiva, en economías en desarrollo como las de América del Sur, cuya agenda comercial se basa en la exportación de productos de bajo valor agregado y en la importación de productos de alto valor agregado, el impacto del efecto derrame de crecimiento de un socio comercial importante como China puede ser negativo. Vale decir, pese al aumento del valor total exportado por América del Sur hacia China, el incremento de las importaciones sudamericanas de productos procedentes de este país puede ser todavía mayor como consecuencia de una mayor competitividad de los productos chinos, dado que la competitividad de estos productos está parcialmente ligada al crecimiento (desarrollo) económico, es decir, a las mejoras en el proceso productivo.

Los efectos del comercio internacional sobre una economía son, en términos generales, heterogéneos (Kneller, Morgan y Kanchanahatakij, 2008). Desde esta perspectiva, cabe destacar que existen en la literatura dos vertientes teóricas en relación con el impacto de los intercambios internacionales. La primera, conocida como crecimiento impulsado por las exportaciones (export-led growth) ${ }^{3}$, destaca que el crecimiento de las exportaciones ejerce un efecto positivo sobre el crecimiento de un país. En esta línea, Cândido y Lima (2010) sostienen que las exportaciones tuvieron un papel importante y significativo en el desempeño de algunas economías asiáticas entre 1995 y 2005. Por su parte, Lawrence y Weinstein (1999) señalan que las importaciones fueron uno de los principales factores determinantes para el crecimiento del Japón en el período 1964-1973. Por su parte, Acharya y Keller (2008) destacan además que las importaciones constituyen una importante fuente de aprendizaje tecnológico para un país.

\footnotetext{
3 Línea de pensamiento que destaca el crecimiento de las exportaciones como principal determinante del crecimiento de la renta de un país.
} 
La segunda línea de pensamiento subraya la posibilidad de que la apertura comercial tenga un impacto negativo sobre el crecimiento económico. De acuerdo con este planteamiento, el comercio exterior puede provocar una reducción de la participación de la industria en el porcentaje de empleo y del PIB de una economía determinada (Bresser-Pereira y Marconi, 2010; Nassif, 2008; Oreiro y Feijó, 2010).

Pese a que en el corto plazo el aumento de las importaciones pueda tener un impacto negativo sobre la economía, a largo plazo puede incidir positivamente sobre su tasa de crecimiento. A partir del modelo teórico elaborado por Grossman y Helpman (1997), una economía se puede beneficiar de las importaciones de productos y servicios ya que por medio de ellas podrá ampliar su acervo de conocimientos. En otras palabras, los autores destacan que las importaciones influyen de manera positiva en el progreso tecnológico interno.

Este progreso, tal y como se destaca en el modelo neoclásico de crecimiento de Solow, resulta fundamental para el crecimiento a largo plazo de una economía (Romer, 2011). El modelo elaborado por Solow destaca el progreso tecnológico como variable exógena, pero, desde la perspectiva que destacan Grossman y Helpman (1997), este podrá desarrollarse internamente o podrá "importarse" desde otros países. Tal proceso de "importación de tecnología" se conoce como derrame tecnológico y podría considerarse una subdivisión del derrame de crecimiento. De acuerdo con Keller (2009) y Grossman y Helpman (1997), el trasvase tecnológico (derrame) de una economía a otra se produce principalmente por los intercambios internacionales y, en concreto, por la importación de productos y servicios.

Así pues, por todo lo expuesto, es importante considerar el efecto conjunto de las importaciones y las exportaciones en el desempeño económico. De acuerdo con la teoría desarrollada por Kaldor (las conocidas leyes de Kaldor) tanto la expansión de las exportaciones como de las importaciones deben producirse de modo que no haya desequilibrios en la balanza de pagos (Lamonica y Feijó, 2011).

A partir de las leyes de Kaldor, Thirlwall y Hussain (1982) elaboraron un modelo teórico en el que el crecimiento de una economía se puede explicar parcialmente por la demanda internacional de productos, vale decir, por el equilibrio de la balanza comercial. Según los autores, el principal obstáculo al crecimiento a largo plazo de las economías en desarrollo es el desequilibrio en la balanza de pagos, resultante sobre todo de la balanza comercial.

Según se ha indicado, ya sea por las relaciones de oferta y demanda o por la transferencia tecnológica, se puede afirmar que el crecimiento de un país tendrá un impacto sobre el crecimiento de otro país. Aun así, el efecto final de esa relación dependerá del patrón comercial existente entre los países.

Además, suponiendo que exista un equilibrio entre las exportaciones y las importaciones, el impacto de la expansión de la demanda en la tasa de crecimiento de un país a través del aumento de las exportaciones vendrá determinado por el conjunto de productos exportados. Por otra parte, tal y como pone de manifiesto la teoría del desarrollo de North (1977), la diversificación de la cartera exportadora de un país o región resulta de vital importancia para determinar su desarrollo y su crecimiento. Básicamente, en una economía cuya cartera de exportación está poco diversificada e incluye productos de bajo valor agregado, el crecimiento de las exportaciones se verá restringido a unos pocos sectores. En consecuencia, el efecto del aumento de las exportaciones en el crecimiento de la economía será escaso. 


\section{Metodología}

\section{Efecto derrame del crecimiento basado en el comercio internacional}

Para determinar el efecto derrame del crecimiento de China sobre la tasa de crecimiento sudamericana a partir de las relaciones comerciales existentes entre ambas regiones, se recurrió a un modelo econométrico dinámico para datos de panel. La dinámica fue introducida en el modelo a través de la inclusión de la variable dependiente desfasada (Greene, 2002).

Si bien según Gomes y Braga (2008) el uso de modelos dinámicos con datos de panel aporta beneficios tales como el aumento de grados de libertad o la reducción de la multicolinealidad existente entre las variables independientes, además de una mayor eficacia en la estimación de coeficientes este procedimiento lleva aparejadas complicaciones en el análisis. La presencia en el modelo de la variable dependiente desfasada genera una correlación de las variables independientes con el término de error, concretamente de la variable dependiente desfasada con el error. Esta correlación, denominada correlación en serie, provoca problemas de endogeneidad entre las variables (Greene, 2002).

Para corregir estos problemas se suele recurrir a variables instrumentales como la estimación de los coeficientes por medio del método de los momentos generalizado (GMM). En concreto, el panel dinámico se podría estimar empleando el método elaborado por Arellano y Bond (1991). Este método consiste en estimar la ecuación utilizando variables instrumentales correlacionadas con las variables independientes, pero no con el error. Estos instrumentos se pueden obtener por medio de la propia variable desfasada o de la diferencia desfasada para encontrar estimadores más eficaces, de modo que la heterogeneidad no observada en el modelo, $v_{i}$, se elimine (Gomes, 2007; Greene, 2002). Por consiguiente, en este trabajo se utilizó el método de los momentos generalizado diferenciado (GMM-dif).

Dicho esto, el modelo que se va a estimar se puede expresar de la siguiente manera:

$$
\begin{aligned}
& \text { pib }_{i, t}=\beta_{0}+\beta_{1} \text { pib }_{i, t-1}+\beta_{2} \text { términosT }_{i, t}+\beta_{3} \text { est_r_capitales }_{i, t}+\beta_{4} \text { pib_CH }_{t} \\
& +\beta_{5} \text { pib_CH01 }{ }_{t}+\beta_{6} \text { año01 }+\beta_{7} \text { año08 }+u_{i, t}
\end{aligned}
$$

donde pib $_{i, t}$ representa la tasa de crecimiento del país $i$ (Argentina, Brasil, Colombia y Chile, es decir $i=1,2,3$ y 4 ) en el período $t$ (para $t=1, \ldots, T$ ); $p i b_{i, t-1}$, la tasa se crecimiento de la renta desfasada en un período de tiempo; términos $T_{i, t}$ la tasa de crecimiento de los términos de intercambio del país $i$ en relación con China; est_r_capitales ${ }_{i, t}$ representa la tasa de crecimiento de las reservas reales de capital extranjero en el país $i$; $p i b_{-} \mathrm{CH}_{t}$ la tasa de crecimiento de la renta interna de China; y $u_{i, t}$ representa el término de error de la ecuación. Además, se incluyen variables ficticias en forma mixta con objeto de captar los efectos del cambio del comercio exterior entre estos países en 2001 y desde 2008. En la variable año01 se atribuye el valor 0 a los años anteriores a 2001 y el valor 1 para los demás. Del mismo modo, en el caso de la variable año08 se atribuye el valor 0 a los años anteriores a 2008, y el valor 1 para los demás años.

La variable ficticia aditiva año01 se incluye en el modelo para controlar el efecto que tuvo sobre la tasa de crecimiento autónomo de los países sudamericanos la expansión generalizada del comercio de América del Sur con el resto del mundo que se produjo en 2001. La variable ficticia aditiva año08 tiene por objeto controlar el efecto de la crisis financiera internacional que tuvo su origen en los Estados Unidos en 2008. Por último, la variable $\mathrm{pib} \_\mathrm{CH} 01_{t}$ se incluye para captar el efecto que tuvo sobre el derrame del crecimiento chino el aumento de la participación de este país en el comercio sudamericano a partir de 2001. Esta variable representa la interacción entre la variable ficticia año01 y pib_CH${ }_{t}$. 
Es importante destacar una vez más que por medio de la metodología de Arellano y Bond (1991) se elimina la endogeneidad entre los precios internos, los externos y el tipo de cambio, así como la simultaneidad existente entre el crecimiento de la renta externa y de la renta interna.

El modelo expresado en la ecuación (1) se basa en el modelo teórico elaborado por Thirlwall y Hussain (1982). De acuerdo con este modelo, el crecimiento a largo plazo de una economía en desarrollo viene determinado en parte por la relación entre las exportaciones, las importaciones y por el flujo de capitales. Esta relación se puede expresar del siguiente modo:

$$
\left(\frac{E}{R}\right)\left(p_{d t}+x_{t}\right)+\left(\frac{C}{R}\right)\left(c_{t}\right)=p_{f t}+m_{t}+e_{t}
$$

En la ecuación (2) se expresa la relación entre la tasa de crecimiento del flujo de capitales $\left(c_{t}\right)$, la proporción de las importaciones financiadas por los ingresos procedentes de las exportaciones $(E / R)$ y la proporción financiada por el flujo de capitales $(\mathrm{C} / \mathrm{R})$, además de las tasas de crecimiento de las exportaciones $\left(x_{t}\right)$, de los precios internos $\left(p_{d t}\right)$, de los precios externos $\left(p_{f t}\right)$, de las importaciones $\left(m_{t}\right)$ y, además, de la tasa de crecimiento del tipo de cambio $\left(e_{t}\right)$. Ambas proporciones se utilizan para ponderar la participación de las exportaciones y del flujo de capitales, teniendo en cuenta que poseen un peso diferente por lo que respecta al equilibrio de la balanza de pagos (Thirlwall y Hussain, 1982).

De acuerdo con el modelo de Thirlwall y Hussain (1982), las tasas de crecimiento de las exportaciones y de la importación se pueden representar como:

$$
\begin{aligned}
m_{t} & =\psi\left(p_{f t}-e_{t}-p_{d t}\right)+\pi\left(y_{t}\right) \\
x_{t} & =\eta\left(p_{d t}-e_{t}-p_{f t}\right)+\epsilon\left(z_{t}\right)
\end{aligned}
$$

donde $\psi$ representa la elasticidad precio de la demanda por importaciones; $\pi$, la elasticidad renta de la demanda por importaciones; $\eta$, la elasticidad precio de la demanda por exportaciones; $\epsilon$, la elasticidad renta de la demanda por exportaciones; $y_{t}$, la tasa de crecimiento de la renta interna, y $z_{t}$, la tasa de crecimiento mundial (socio comercial). De acuerdo con el modelo antes mencionado, se espera que: $\psi<0 ; \pi>0 ; \eta<0 ; e>0$.

Sobre la base de todo lo expuesto, se puede definir una relación clara entre la tasa de crecimiento de un país y la de otro por medio de las tasas de crecimiento de las exportaciones y las importaciones. Esta relación se determina como:

$$
y_{t}=\frac{\left(\frac{E}{R} \eta+\psi+1\right)\left(p_{d t}-e_{t}-p_{f t}\right)+\frac{E}{R}\left(\epsilon\left(z_{t}\right)\right)+\frac{C}{R}\left(c_{t}-p_{d t}\right)}{\pi}
$$

En la ecuación (1), la variable termos $T_{i, t}$ representa la relación $\left(\mathrm{p}_{\mathrm{dt}}-\mathrm{e}_{\mathrm{t}}-\mathrm{p}_{\mathrm{ft}}\right)$, mientras que est_r_capitales ${ }_{i, t}$ representa la relación $\left(c_{t}-p_{d t}\right)$ y la variable $p i b_{-} C H_{t}$ representa la variable $z_{t}$.

Así pues, se espera que el efecto de inercia de la tasa de crecimiento de la renta $\beta_{1}$ sea positivo. Por lo que respecta al efecto de la tasa de crecimiento de los términos de intercambio, se espera que tenga una correlación positiva con la variable dependiente, puesto que una mejora en los términos de intercambio llevaría aparejado un aumento relativo del valor agregado exportado por los países de América del Sur y, por consiguiente, un aumento de su productividad interna. Con todo, cabe destacar que este efecto depende de la relación entre la elasticidad precio de la demanda por exportaciones $\eta$, la elasticidad precio de la demanda por importaciones , $\psi$, y la elasticidad renta de la demanda por importaciones $\pi$, así como de la proporción de importaciones financiadas por las exportaciones $E / R$. 
Al igual que en el caso anterior, se espera que la tasa de crecimiento de las reservas reales de capital extranjero est_r_capitales ${ }_{i, t}$, presente signo positivo en relación con la variable dependiente analizada. Esto es así porque, tal y como destacan Thirlwall y Hussain (1982) y Laplane y Sarti (1997), las inversiones extranjeras directas cumplen un papel muy importante a la hora de financiar el crecimiento de los países en desarrollo.

Por último, asumiendo la hipótesis de que el efecto derrame del crecimiento de China tenga un impacto positivo sobre el crecimiento de los países de América del Sur, se espera que el efecto final del aumento de la tasa de crecimiento de la renta de los polos comerciales sea positivo.

\section{Origen de los datos}

Los datos utilizados en este trabajo proceden de fuentes secundarias nacionales e internacionales y comprenden los cuatro países de América del Sur en cuestión (Argentina, Brasil, Chile y Colombia), además de China. Las fuentes consultadas fueron la Organización Mundial del Comercio (OMC), el Departamento de Agricultura de los Estados Unidos y la Conferencia de las Naciones Unidas sobre Comercio y Desarrollo (UNCTAD).

Los datos referentes a la variación anual del tipo real de cambio y a la tasa de crecimiento de los índices de precios al consumo de cada país (Argentina, Brasil, Chile, China y Colombia) corresponden al año de referencia 2010 y se obtuvieron de la base de datos del USDA. Por último, la tasa de crecimiento del producto interno bruto de los países analizados en este estudio, los valores corrientes referentes al flujo y a las reservas de capital extranjero en los países de América del Sur, así como a los valores de los índices de complementariedad y similitud de las exportaciones de los países sudamericanos, proceden de la base de datos de la UNCTAD.

\section{Resultados y discusiones}

Dado el carácter provisional de los datos utilizados en las estimaciones de los paneles dinámicos, en todas las variables utilizadas se aplicaron las pruebas de raíz unitaria de Levin-Lin-Chu (LLC), Harris-Tzavalis (HT), Breitung e Im-Pesaran-Shin (IPS). En el período estudiado solo se constató la presencia de raíz unitaria en la tasa de crecimiento del nivel de precios en los países sudamericanos. El problema de la no estacionariedad de esta serie se eliminó tomando la primera diferencia de la variable. A continuación, se crearon las variables que representan los términos de intercambio de los países sudamericanos con China, así como la variable que representa las reservas reales de capital extranjero en los países sudamericanos, conforme a lo señalado en la metodología del presente trabajo. Volvieron a aplicarse las pruebas de raíz unitaria sin que se indicara la presencia de la misma.

Por último, para verificar si el modelo estimado presentaba un buen ajuste, se realizó la prueba de autocorrelación de Arellano y Bond, que se aplica a los residuos en diferencia del modelo y pretende probar la ausencia de correlación en serie de primer y segundo orden. Según Silva (2014), debe rechazarse la hipótesis de ausencia de autocorrelación en serie de primer orden, pero no la de segundo orden. En el caso analizado, la hipótesis nula de autocorrelación de primer orden fue rechazada con un nivel de significancia del 10\%, si bien por otra parte no fue posible rechazar la hipótesis nula en la autocorrelación de segundo orden con ningún nivel de significancia. Esta prueba ha permitido constatar que los parámetros estimados son robustos y que los valores observados tienden a acercarse a los valores reales.

En el cuadro 1 se presentan los resultados de la estimación del efecto derrame del crecimiento chino sobre la tasa de crecimiento de los países sudamericanos. La primera variable que figura en el 
cuadro hace referencia a la tasa de crecimiento de los países sudamericanos desfasada en un período. Esta variable resultó estadísticamente significativa con un nivel del 1\% de significancia. Además, presentó una relación positiva con la tasa de crecimiento de los países sudamericanos. Teniendo en cuenta que esta variable representa la inercia del crecimiento del PIB, este resultado se ajusta a la teoría, ya que es de prever que el crecimiento de la economía en el año anterior influya sobre el crecimiento actual del PIB y que esta correlación causal sea positiva. Se observa además que, según el modelo, el aumento de un punto porcentual en el crecimiento pasado elevará el crecimiento futuro del PIB en aproximadamente 0,18 puntos porcentuales.

\section{Cuadro 1}

Efecto derrame del crecimiento de China sobre el crecimiento de los países sudamericanos, 1981-2014

\begin{tabular}{lcccc}
\hline Variables & Coeficiente & Errores estándar robustos & Estadística Z & p-valor \\
\hline pib ${ }_{t-1}$ & 0,1833 & 0,0504 & 3,63 & $0,000^{*}$ \\
\hline TérminosT_CH & 0,0483 & 0,0073 & 6,59 & $0,000^{*}$ \\
\hline est_r_capitales & 0,0496 & 0,0067 & 7,31 & $0,000^{*}$ \\
\hline pib_CH & 0,2298 & 0,0948 & 2,42 & $0,015^{\star *}$ \\
\hline pib_CH01 & 0,4744 & 0,3724 & 1,27 & $0,203 N S$ \\
\hline año01 & $-4,7709$ & 3,5061 & $-1,36$ & $0,174 N S$ \\
\hline año08 & 0,7278 & 0,6043 & 1,20 & 0,229 NS \\
\hline constante & 0,8928 & 0,9262 & 0,96 & $0,335 N S$ \\
\hline Número de observaciones: 128 & & Wald chi2(3) $=63,73$ & & Prob $>$ chi2 $=0,000$ \\
\hline
\end{tabular}

Fuente: Elaboración propia.

Nota: $\quad$ * significativo a $1 \%$; ** significativo a $5 \%$; NS: no significativo.

Variables: $p i b_{t-1}$ es la tasa de crecimiento de los países sudamericanos desfasada para un período; términos $T_{-} C H$ es la tasa de crecimiento de los términos de intercambio comercial entre los países de América del Sur y China; est_r capitales es la variación anual de las reservas reales de capital extranjero en los países sudamericanos; pib_CH es la tasa de crecimiento de la renta de China; $p i b \_C H 01$ es la variable ficticia de interacción entre pib_CH y año 01 ; año01 es la variable ficticia aditiva que pretende medir los cambios estructurales a partir de 2001; y año08 es la variable ficticia aditiva que pretende medir los cambios estructurales a partir de 2008.

Conforme a lo previsto, la tasa de crecimiento de los términos de intercambio comercial entre los países de América del Sur y China presentó una relación positiva y significativa del 1\% de significancia en el período 1981-2014. Así pues, el aumento de 1 punto porcentual en la variable términosT_CH conlleva un incremento de aproximadamente 0,048 puntos porcentuales en la tasa media de crecimiento de los países sudamericanos. Teniendo en cuenta que esta variable representa la relación entre los precios de los productos exportados por los países sudamericanos y los precios de los productos chinos importados por dichos países, el valor relativamente bajo que presenta el coeficiente estimado de esta variable se ajusta a lo esperado, ya que las transacciones de los países de América del Sur con sus socios comerciales se centran principalmente en la exportación de productos de bajo valor agregado (escaso contenido tecnológico-productos básicos) y en la importación de productos de elevado valor agregado (Cunha, 2011).

En concreto, se puede tomar como ejemplo el comercio del Brasil (principal representante de América del Sur por lo que respecta a flujo comercial y PIB) con China. Históricamente, esta relación se caracteriza por la expansión de las exportaciones brasileñas hacia China de productos de escaso valor agregado, mientras que en contrapartida se produce un aumento en la importación de productos con un mayor valor agregado (Mattos y Carcanholo, 2012). Además, cabe destacar también que una de las características principales de los productos de escaso valor agregado (productos básicos) es el bajo poder de repercusión que su producción tiene en la economía interna en comparación con los demás productos/sectores, dado que su producción presenta un reducido poder de encadenamiento 
hacia adelante o hacia atrás. Por consiguiente, si la agenda comercial sudamericana con China se mantiene sin cambios, aunque se produzca una mejora en los términos de intercambio seguida de un aumento de la producción en los sectores beneficiados, su efecto final sobre el crecimiento de las economías sudamericanas será escaso, tal y como se ilustra en el cuadro 1.

Por su parte, la variable est_r_capitales, que expresa el efecto de las reservas reales de capital extranjero en los países sudamericanos, resultó significativa en un nivel del 1\% y con signo positivo. Este resultado es acorde con lo que se esperaba, dado que las reservas de capitales resultan fundamentales para el desarrollo de los países sudamericanos o, en general, de los países en desarrollo, como consecuencia de la importancia de dichas reservas para financiar los déficits en la balanza de pagos (Thirlwall y Hussain, 1982).

Con todo, por el coeficiente estimado se nota un efecto relativamente bajo de esta variable en la tasa de crecimiento sudamericano, puesto que el aumento de 1 punto porcentual en la variación anual de estas reservas de capital conllevará un aumento aproximado de 0,049 puntos porcentuales. Teniendo en cuenta la importancia de la inversión extranjera directa para las economías en desarrollo (periféricas y semiperiféricas), se preveía que la variable est_r_capitales ejerciera una mayor influencia sobre la tasa de crecimiento de los países sudamericanos, tal y como destacan Thirlwall y Hussain (1982), Laplane y Sarti (1997) y Aoun, Verdi y Sato (2008).

El reducido impacto de la variable est_r_capitales se debe posiblemente a la influencia que sobre su determinación ejerce la inflación de los países sudamericanos. Por definición, dicha variable viene determinada por la diferencia entre la variación anual de las reservas de capital en valores corrientes y por la tasa de crecimiento del índice de precios de los países sudamericanos. Teniendo en cuenta que, según Bandeira (2002), la inflación de los países sudamericanos ha sido tradicionalmente elevada, el impacto de las reservas reales de capital tenderá a ser limitado.

Por lo que respecta a la variable $\mathrm{pib}_{-} \mathrm{CH}$, que representa la tasa de crecimiento de la renta china y, por consiguiente, el coeficiente del efecto derrame del crecimiento de China sobre la tasa de crecimiento de los países sudamericanos, de acuerdo con el cuadro 1 se percibe que el coeficiente estimado es estadísticamente significativo: presenta un nivel de significancia del $5 \%$ y tiene una correlación positiva con la tasa de crecimiento de estos países. Este resultado se ajusta a la hipótesis inicial del trabajo y corrobora la idea de la importancia del crecimiento de China para los países sudamericanos puesto que, si las demás condiciones permanecen constantes, este crecimiento conllevaría el crecimiento del subcontinente.

A partir de lo expuesto en la introducción y en la revisión de literatura del presente trabajo, este efecto derrame del crecimiento chino tendría una influencia positiva sobre la tasa de crecimiento sudamericana por dos motivos: primero, por el aumento de la demanda china de productos sudamericanos, como se indica en el modelo teórico elaborado por Thirlwall y Hussain (1982). Segundo, por el acceso que los países sudamericanos han tenido al acervo de conocimientos de China por medio de la importación de bienes y servicios, tal como destacan Grossman y Helpman (1997).

En concreto, de las variables incluidas en este primer análisis, esta es la que genera un mayor impacto sobre la tasa de crecimiento de los países sudamericanos, ya que un incremento de 1 punto porcentual en la tasa de crecimiento de China conllevará un incremento aproximado de 0,23 puntos porcentuales en el crecimiento de los países sudamericanos. Dicho de otro modo: con esta variable se puede reafirmar la importancia del crecimiento chino para los países de América del Sur.

La variable ficticia interactiva, pib_CHO1, incluida para verificar si hubo cambios en el efecto derrame del crecimiento chino sobre la tasa de crecimiento sudamericana a partir de 2001, no fue estadísticamente significativa. Este resultado sugiere que el aumento del flujo comercial entre los 
países de América del Sur y China no alteró de manera significativa el efecto derrame del crecimiento chino sobre la tasa de crecimiento sudamericana. Así pues, se observa que el volumen del flujo comercial entre dos países no es el principal determinante del efecto derrame.

Este resultado es acorde con el modelo teórico utilizado en el presente trabajo. Según el modelo de Thirlwall (1979), precursor del modelo que se sigue en este trabajo, la tasa de crecimiento de una economía es aproximadamente igual a la tasa de crecimiento de las exportaciones, dividida por la elasticidad renta de las importaciones. De este modo, para que una economía presente mayores tasas de crecimiento a largo plazo, debe haber cambios en el patrón de importaciones (en la elasticidad renta de las importaciones), además de un aumento en las exportaciones de productos. Sobre la base de lo presentado en la introducción del presente trabajo se puede inferir que ambos crecieron en proporciones prácticamente iguales entre 2001 y 2014, lo cual implica un efecto mínimo del crecimiento de las exportaciones sobre la tasa de crecimiento de los países sudamericanos.

Dicho de otro modo, las exportaciones de productos sudamericanos hacia China han aumentado de manera casi exponencial a partir de 2001. Este aumento tendría una influencia positiva sobre la tasa de crecimiento de los países sudamericanos, lo que se debería al hecho de que el incremento de las exportaciones en el período podría estar incentivando la productividad interna, tal como destacan autores de la corriente de crecimiento impulsado por las exportaciones como Edwards (1992), Melitz (2003) y Wacziarg y Welch (2008), quienes sostienen que el crecimiento de las exportaciones (o del comercio en general) cumple un papel fundamental en el aumento de la tasa de crecimiento interno.

Sin embargo, el aumento del volumen de las exportaciones de productos sudamericanos hacia China ha venido acompañado del aumento de las importaciones de productos chinos por parte de estos países, lo que, por su parte, puede haber desincentivado la productividad interna. Este posible efecto negativo sobre la productividad interna remite parcialmente al llamado proceso de desindustrialización de la economía que destacan Bresser-Pereira y Marconi (2010), Nassif (2008) y Oreiro y Feijó (2010). Dicho esto, se plantea la hipótesis de que la interacción de estos dos efectos podría anular el aumento significativo de las exportaciones sudamericanas hacia China o, incluso, reducir los efectos positivos de ese incremento.

Por otra parte, es importante tener en cuenta la diversidad de la cartera de exportaciones sudamericana. Como resalta North (1977), la diversificación de la cartera exportadora de una región resulta fundamental para calcular el impacto del sector exportador en la determinación de la renta de la región. Por consiguiente, una región con una cartera de exportaciones poco diversificada obtendrá ganancias reducidas de una expansión de las exportaciones. Además, puesto que en el caso sudamericano se trata de una cartera exportadora con bajo valor agregado, la repercusión que tiene el aumento de dichas exportaciones en la economía es bastante restringida.

En el cuadro 2 se puede comprobar que la diversidad de la cartera exportadora sudamericana es pequeña en comparación con el alcance que podría tener. El índice de complementariedad, que figura en el cuadro 2, pone en evidencia la escasa capacidad de las exportaciones sudamericanas para atender la demanda china de importaciones.

En contrapartida, en el cuadro 2 se observa la mayor capacidad de China para hacer frente a la demanda de importaciones de los países sudamericanos. En promedio, se aprecia que la cartera exportadora sudamericana atiende solo al $29,1 \%$ del conjunto de la demanda china. Frente a esto, China consigue atender de media el 44,9\% de la demanda de los países de América del Sur. Queda patente que el alcance que tienen los productos chinos en los mercados sudamericanos es mayor que el que tienen los productos sudamericanos en los mercados chinos. 
Cuadro 2

Índice de complementariedad

\begin{tabular}{lcc}
\hline Año & China-América del Sur & América del Sur-China \\
\hline 1995 & 0,418 & 0,276 \\
\hline 1996 & 0,419 & 0,267 \\
\hline 1997 & 0,427 & 0,281 \\
\hline 1998 & 0,428 & 0,271 \\
\hline 2099 & 0,434 & 0,275 \\
\hline 2001 & 0,456 & 0,300 \\
\hline 2002 & 0,456 & 0,297 \\
\hline 2003 & 0,417 & 0,284 \\
\hline 2004 & 0,421 & 0,282 \\
\hline 2005 & 0,433 & 0,285 \\
\hline 2007 & 0,454 & 0,288 \\
\hline 2008 & 0,466 & 0,293 \\
\hline 2009 & 0,455 & 0,290 \\
\hline 2010 & 0,475 & 0,313 \\
\hline 2011 & 0,471 & 0,307 \\
\hline 2012 & 0,476 & 0,303 \\
\hline Media & 0,473 & 0,307 \\
\hline & 0,474 & 0,307 \\
\hline
\end{tabular}

Fuente: Conferencia de las Naciones Unidas sobre Comercio y Desarrollo (UNCTAD), "Data Center", 2015 [en línea] http:// unctadstat.unctad.org.

Además de lo ya expuesto, cabe destacar que el efecto final de este derrame está directamente relacionado con la elasticidad renta de la demanda por exportaciones $\epsilon$, la elasticidad renta de la demanda por importaciones $\pi$, y el porcentaje de importaciones financiado por los ingresos provenientes de las exportaciones. Por un lado, un aumento de $\pi$, reducirá el efecto derrame chino; por otro lado, un aumento de $\epsilon$ y del porcentaje de las importaciones financiado mediante los ingresos de las exportaciones aumentará dicho derrame. En concreto, es posible deducir que el aumento de las importaciones de productos chinos por parte de los países de América del Sur viene parcialmente determinado por el aumento de la elasticidad renta de la demanda por importaciones y que este aumento conlleva la anulación del aumento del porcentaje de las importaciones financiado mediante los ingresos provenientes de las exportaciones destinadas a China, así como de la elasticidad renta de la demanda por exportaciones.

De este modo, teniendo en cuenta que el efecto derrame chino depende de la elasticidad renta de la demanda por exportaciones y esta a su vez viene determinada en parte por la complementariedad de las exportaciones sudamericanas con las importaciones chinas, su aumento en las economías sudamericanas se producirá gracias a una mayor participación de los productos sudamericanos en las importaciones chinas. No obstante, de acuerdo con el cuadro 2 no se produjo ningún cambio significativo del índice de complementariedad entre estos países, lo que explica la estabilidad del efecto derrame del crecimiento de China.

Esta estabilidad puede reflejar, por un lado, la falta de políticas públicas que den lugar a una mayor diversificación de la cartera de exportaciones sudamericanas hacia China, de modo que este aumento atienda a la demanda de productos importados por este país y, por otro lado, una incapacidad de inserción de los productores sudamericanos en los mercados chinos. En parte, la incapacidad de América del Sur para penetrar en los mercados chinos está ligada directamente a la realidad interna de ese país. De acuerdo con el estudio de Filgueiras y Kume (2010), se constata que la fuerte competitividad china se explica por la baja calidad de los productos y, por consiguiente, al 
menor precio. Además, Schott (2006) menciona como otro posible motivo de la alta competitividad de China los bajos salarios que allí se perciben.

Por último, aunque se hayan incluido variables ficticias aditivas (año01 y año08) a fin de medir un posible cambio estructural en la tasa media de crecimiento de los países sudamericanos, se percibe que ninguna de ellas resultó significativa. De este modo, ni la expansión del flujo comercial en 2001 ni la crisis de 2008 tuvieron una influencia directa sobre la tasa de crecimiento de estos países. Además de estas variables, la constante expresada en el modelo tampoco fue significativa para el análisis.

Pese a que muchos trabajos han destacado la importancia de China para el comercio sudamericano a partir de 2001, como por ejemplo Cunha (2007 y 2011) y Crossetti y Fernandes (2005), en este trabajo se demuestra que no se produjo ninguna alteración estadísticamente significativa del efecto derrame chino sobre la tasa media de crecimiento de los países sudamericanos. Por lo tanto, aunque China se haya convertido en una importante fuente de derrame del crecimiento por su elevada demanda de productos básicos, tal y como destacan Poirson y Weber (2011), su impacto sobre la tasa de crecimiento de los países sudamericanos no ha presentado ninguna alteración significativa en los últimos años.

Por lo que respecta a sus relaciones comerciales con América del Sur, es importante destacar que China ejerce una gran presión sobre las industrias locales dada su presencia en mercados importantes para el desarrollo industrial (Mattos y Carcanholo, 2012). Por ende, el crecimiento de la economía china tiene también un impacto indeseado sobre el crecimiento de los países sudamericanos.

Por último, se concluye que las estimaciones que figuran en el cuadro 1 son estadísticamente significativas y acordes con las hipótesis del presente trabajo. No obstante, cabe destacar que, aunque el efecto derrame del crecimiento chino tenga una correlación positiva con el crecimiento de los países sudamericanos, no se ha visto influido por el significativo aumento del flujo comercial ente China y América del Sur a partir de 2001.

\section{Consideraciones finales}

Dado que el crecimiento de China ha ejercido una influencia positiva sobre el flujo comercial del país con las economías sudamericanas, sobre todo en los últimos 10 años, el presente trabajo ha pretendido analizar el efecto derrame del crecimiento chino sobre las tasas de crecimiento de los países de América del Sur. Se ha prestado especial atención al impacto de China después de 2001, puesto que fue en este período cuando el flujo comercial entre dichos países presentó sus mayores variaciones. En especial, sobre la base de las teorías abordadas en el presente trabajo, se ha destacado que el impacto del crecimiento de China sobre la tasa de crecimiento de los países sudamericanos tiene como principal canal de propagación los intercambios comerciales entre estos países.

Tal y como se esperaba, se ha observado que el crecimiento de China ha supuesto un impacto positivo e importante sobre el crecimiento de los países sudamericanos en el período 1981-2014. Por lo tanto, tomando como base los modelos de demanda internacional, se puede afirmar que la demanda china de productos sudamericanos influye de manera positiva sobre la productividad de estos países. Así pues, esta relación convierte al crecimiento de China o, más concretamente, de la demanda china, en un importante factor para el crecimiento de los países de América del Sur, a pesar de su carácter exógeno con respecto a las economías del continente.

Además, visto que el flujo comercial entre China y los países de América del Sur presentó un gran aumento en 2001, se ha pretendido analizar el impacto que este incremento tendría sobre el efecto derrame del crecimiento chino. No obstante, se ha comprobado que no se ha producido un cambio estadísticamente significativo de este efecto a partir de 2001. También cabe destacar 
que en el período analizado no se ha producido ningún cambio de importancia relativa en el índice de complementariedad de las exportaciones sudamericanas con respecto a las importaciones chinas. Dicho de otro modo, no se ha producido ningún aumento de la diversidad de la cartera de exportaciones sudamericanas hacia China, a pesar de que según el índice de complementariedad existiera la posibilidad de aumentar el número de productos exportados por estos países.

Así pues, se puede afirmar que la simple expansión de las exportaciones de los sectores tradicionales (productos básicos) hacia China no es suficiente para afectar al derrame del crecimiento sobre estos países a partir de las relaciones comerciales. En este sentido, cabe destacar la importancia de adoptar políticas que aumenten la diversificación de la cartera de exportaciones de América del Sur hacia China, ya sea a través del mantenimiento de los precios, de la facilitación de las exportaciones, o de una inversión en infraestructura que posibilite un incremento de la competitividad de los productos sudamericanos en el mercado internacional.

De este modo, aunque exista una relación positiva entre el crecimiento de China y el crecimiento de las principales economías de América del Sur, es preciso adoptar políticas que amplíen la cartera de exportaciones sudamericanas hacia China. Esta ampliación resulta de suma importancia para la expansión del efecto derrame del crecimiento chino sobre el crecimiento sudamericano. Tal y como se ha observado, el crecimiento de China ha resultado destacado incluso entre países con un desarrollo similar.

Pese a las dificultades derivadas de competir directamente con estos países debido a la alta competitividad, la ampliación del efecto derrame a través de un fortalecimiento de las relaciones comerciales en varios sectores constituye una importante vía de crecimiento. No obstante, se ha aprovechado poco el rápido crecimiento de China.

Por último, pese a la importancia de ampliar la cartera de exportaciones sudamericanas hacia China, el presente trabajo no ha podido detallar este hecho. Por lo tanto, siguen siendo necesarias nuevas investigaciones que indiquen cuáles de entre los potenciales mercados chinos garantizarán mayores ganancias. Dicho de otro modo: cuáles de los productos demandados por China pueden ser exportados por las economías sudamericanas. Además, es importante destacar qué mercados sudamericanos se han visto influidos negativamente por la presencia china.

\section{Bibliografía}

Acharya, R. C. y W. Keller (2008), "Estimating the productivity selection and technology spillover effects of imports", NBER Working Paper, N 14079, Cambridge, Massachusetts, Oficina Nacional de Investigaciones Económicas.

Aoun, S., A. R. Verdi y G. S. Sato (2008), "Dinâmica das fusões e aquisições no investimento direto estrangeiro: especificidade da indústria de alimentos e bebidas no Brasil, 1996-2006", Informações Econômicas, vol. 38, № 3, São Paulo, Instituto de Economía Agrícola (IEA).

Arellano, M. y S. Bond (1991), "Some tests of specification for panel data: Monte Carlo evidence and an application to employment equations", The Review of Economic Studies, vol. 58, №2, Oxford University Press.

Bandeira, L. A. M. (2002), "As políticas neoliberais e a crise na América do Sul", Revista Brasileira de Política Internacional, vol. 45, № 2, Brasilia, Instituto Brasileño de Relaciones Internacionales.

Bayoumi, T. y A. Swiston (2009), "Foreign entanglements: estimating the source and size of spillovers across industrial countries", IMF Staff Papers, vol. 56, № 2, Washington, D.C., Fondo Monetario Internacional (FMI).

Bresser-Pereira, L. C. y N. Marconi (2010), "Existe doença holandesa no Brasil?", Doença holandesa e indústria, L. C. Bresser Pereira (org.), Río de Janeiro, Editora FGV.

Cândido, M. S. y F. G. Lima (2010), "Crescimento econômico e comércio exterior: teoria e evidências para algumas economias asiáticas", Revista de Economia Contemporânea, vol. 14, № 2, Río de Janeiro, Instituto de Economía. 
Crossetti, P. A. y P. D. Fernandes (2005), "Para onde vai a China? O impacto do crescimento chinês na siderurgia brasileira", BNDES Setorial, Nㅜ22, Río de Janeiro, Banco Nacional de Desarrollo Económico y Social (BNDES).

Cunha, A. M. (2011), "A China e o Brasil na nova ordem internacional", Revista de Sociologia e Política, vol. 19, Curitiba, Universidad Federal de Paraná.

(2007), "O boom chinês e as economia latino-americanas", Indicadores Econômicos FEE, vol. 35, № 2 , Porto Alegre, Fundación de Economía y Estadística (FEE).

Dornbusch, R. (1976), "Expectations and exchange rate dynamics", The Journal of Political Economy, vol. 84, $N^{\circ}$ 6, Chicago, The University of Chicago Press.

Edwards, S. (1992), "Trade orientation, distortions and growth in developing countries", Journal of Development Economics, vol. 39, № 1, Ámsterdam, Elsevier.

Filgueiras, M. y H. Kume (2010), "A competitividade do Brasil e da China no mercado norte-americano: 2000-2008", Texto para Discussão, № 1501, Brasilia, Instituto de Investigación Económica Aplicada (IPEA).

Gomes, S. C. (2007), "Análise econométrica da produtividade total dos fatores na Amazônia legal, 1990-2004", tesis, Viçosa, Minas Gerais, Universidad Federal de Viçosa.

Gomes, S. C. y M. J. Braga (2008), "Determinantes da produtividade total dos fatores na Amazônia legal: uma aplicação de dados em painel", Amazônia: Ciência \& Desenvolvimento, vol. 3, Banco da Amazônia.

Greene, W. H. (2002), Econometric Analysis, New Jersey, Prentice Hall.

Grossman, G. M. y E. Helpman (1997), "Trade and growth", Innovation and Growth in the Global Economy, Cambridge, Massachusetts, The MIT Press.

Helbling, T. y otros (2007), "Decoupling the train? Spillovers and cycles in the global economy", World Economic Outlook, Washington, D.C., Fondo Monetario Internacional (FMI).

Keller, W. (2009), "International trade, foreign direct investment, and technology spillovers", NBER Working Paper, N ${ }^{\circ} 15442$, Cambridge, Massachusetts, Oficina Nacional de Investigaciones Económicas.

Kneller, R., C. Morgan y S. Kanchanahatakij (2008), "Trade liberalisation and economic growth", The World Economy, vol. 31, № 6, Wiley.

Lamonica, M. T. y C. A. de Feijó (2011), "Crescimento e industrialização no Brasil: uma interpretação à luz das propostas de Kaldor", Revista de Economia Política, vol. 31, № 1, São Paulo.

Laplane, M. y F. Sarti (1997), "Investimento direto estrangeiro e a retomada do crescimento sustentado nos anos 90", Economia e Sociedade, vol. 8, Campinas, Universidad Estadual de Campinas.

Lawrence, R. Z. y D. E. Weinstein (1999), "Trade and growth: import-led or export-led? Evidence from Japan and Korea", NBER Working Paper, № 7264, Cambridge, Massachusetts, Oficina Nacional de Investigaciones Económicas.

Mattos, F. A. M. y M. D. Carcanholo (2012), "Amenazas y oportunidades del comercio brasileño con China: lecciones para Brasil”, Problemas del Desarrollo, vol. 43, № 168, Ciudad de México, Universidad Nacional Autónoma de México (UNAM).

Melitz, M. J. (2003), "The impact of trade on intra-industry reallocations and aggregate industry productivity", Econometrica, vol. 71, № 6, Nueva York, The Econometric Society.

Michaely, M. (1996), "Trade preferential agreements in Latin America: an ex-ante assessment", Policy Research Working Paper, No 1583, Washington, D.C., Banco Mundial.

Nassif, A. (2008), "Há evidências de desindustrialização no Brasil?", Revista de Economia Política, vol. 28, $N^{\circ}$ 1, São Paulo.

North, D. (1977), "Teoria da localização e crescimento econômico regional", Economia regional e urbana: textos escolhidos, J. Schwartzmann (org.), Belo Horizonte, Universidad Federal de Minas Gerais (UFMG).

Obstfeld, M. y K. Rogoff (1997), Foundations of International Macroeconomics, Cambridge, Massachusetts, The MIT Press.

OMC (Organización Mundial del Comercio) (2015), "Estadísticas" [en línea] https://www.wto.org/spanish/ res_s/statis_s/statis_s.htm.

Oreiro, J. L. y C. A. Feijó (2010), "Desindustrialização: conceituação, causas, efeitos e o caso brasileiro", Revista de Economia Política, vol. 30, № 2, São Paulo, abril-junio.

Poirson, H. y S. Weber (2011), "Growth spillover dynamics from crisis to recovery", IMF Working Paper, $N^{\circ}$ WP/11/218, Washington, D.C., Fondo Monetario Internacional (FMI).

Romer, D. (2011), "The Solow growth model", Advanced Macroeconomics, Nueva York, McGraw-Hill. 
Schott, P. K. (2006), "The relative revealed competitiveness of China's exports to the United States vis a vis other countries in Asia, the Caribbean, Latin America and the OECD", Documento de Divulgación ITD, №39, Buenos Aires, Instituto para la Integración de América Latina y el Caribe (INTAL).

Silva, F. A. (2014), "Comércio internacional e crescimento econômico: uma análise considerando os setores e a assimetria de crescimento dos estados brasileiros", Viçosa, Minas Gerais [en línea] http://www.locus. ufv.br/bitstream/handle/123456789/91/texto\%20completo.pdf?sequence=1\&isAllowed=y.

Thirlwall. A. P. (1979), "The balance of payments constraint as an explanation of international growth rates differences", Banca Nazionale del Lavoro Quarterly Review, vol. 32, № 128, Roma, Banca Nazionale del Lavoro.

Thirlwall, A. P. y M. N. Hussain (1982), "The balance of payments constraint, capital flows and growth rate differences between developing countries", Oxford Economic Papers, vol. 34, № 3, Oxford, Oxford University Press.

UNCTAD (Conferencia de las Naciones Unidas sobre Comercio y Desarrollo) (2015) “Data Center” [en línea] http://unctadstat.unctad.org.

Wacziarg, R. y K. H. Welch (2008), "Trade liberalization and growth: new evidence", The World Bank Economic Review, vol. 22, № 2, Washington, D.C., Banco Mundial. 